\title{
Anatomical Overview and Imaging of the Aorta and Visceral Arteries
}

\author{
Gabriele Ironi, Giorgio Brembilla, Giulia Benedetti, \\ and Francesco De Cobelli
}

\subsection{Anatomy}

The thoracic aorta extends proximally from the aortic annulus to the diaphragmatic crura distally. It is subdivided into three parts: the ascending aorta, the arch and the descending aorta. The ascending aorta comprises the aortic root and the tubular ascending aorta. The aortic root lies between the aortic annulus and the sinotubular junction. The sinuses of Valsalva arise from the aortic root. The tubular ascending aorta runs from the sinotubular junction to the brachiocephalic trunk. The coronary arteries are the only branches of the ascending aorta. The aortic arch begins at the brachiocephalic trunk and ends at the origin of the left subclavian artery. The isthmus extends from the left subclavian artery to the ligamentum arteriosum. Three branches usually arise from the aortic arch: the brachiocephalic trunk, the left common carotid artery and the left subclavian artery. The brachiocephalic trunk divides into the right common carotid artery and the right subclavian artery. In $6 \%$ of people, the left

\footnotetext{
G. Ironi $\cdot$ G. Brembilla $\cdot$ G. Benedetti

F. De Cobelli $(\bowtie)$

Department of Radiology and Experimental Imaging Center, San Raffaele Scientific Institute, Milan, Italy

Vita-Salute San Raffaele University, Milan, Italy e-mail: ironi.gabriele@hsr.it; brembilla.giorgio@hsr.it; benedetti.giulia@hsr.it; decobelli.francesco@hsr.it
}

vertebral artery arises directly from the arch [1]. The bovine arch is another variant in which the left common carotid artery arises from the brachiocephalic trunk rather than the aorta [1]. Another arch variant is the ductus diverticulum, a focal bulge along the inner aspect of the isthmus representing a remnant of the ductus arteriosus. Traumatic aortic transection also occurs in this location and can occasionally be difficult to differentiate from a ductus diverticulum. However, the ductus diverticulum has smooth margins with obtuse angles relative to the adjacent aorta. Aortic transection has irregular margins with acute angles relative to the nearby aortic walls.

The descending thoracic aorta extends from the isthmus to the diaphragmatic crura. In contrast to the ascending aorta, the descending thoracic aorta has multiple branches, including the bronchial, intercostal, spinal and superior phrenic arteries and various small mediastinal branches.

The abdominal aorta starts immediately before the emergence of the superior adrenal arteries, approximately at the level of the T12 vertebral body. The juxtarenal abdominal aorta diameter normally measures less than $3 \mathrm{~cm}$. The abdominal aorta supplies visceral and parietal arteries that arise in three vascular planes before it bifurcates to form the common iliac arteries at the L4.

The unpaired visceral arteries to the gastrointestinal tract include the celiac, superior mesenteric and inferior mesenteric arteries. These vessels arise in the midline anterior plane. Shortly 
after its origin, the celiac axis gives rise to three main branches: the left gastric, splenic and common hepatic arteries. The latter subsequently bifurcates into the proper hepatic artery and the gastroduodenal artery.

The paired visceral arteries include the renal, adrenal and gonadal arteries, which run through the lateral plane to supply the urogenital and endocrine abdominal organs. The subcostal, inferior phrenic and lumbar arteries are paired parietal arteries to the diaphragm and body wall, arising in the posterior oblique plane.

As the only unpaired parietal artery, the middle sacral originates posteriorly. The common iliac artery bifurcates at the pelvic brim to yield the hypogastric arteries, which subsequently divide into anterior and posterior branches.

Up to $30 \%$ of subjects have one or more accessory renal arteries on each side that most commonly supply the lower pole, usually arising from the mid-abdominal aorta but occasionally from the distal aorta or common iliac arteries [2,3]. There are also numerous variations of celiac anatomy, most commonly accessory or replaced individual hepatic arteries, with numerous subtypes described in the Michels' classification [4].

\section{$1.2 \quad$ Ultrasound}

Ultrasonographic imaging (US) is characterized by widespread availability, low costs, a lack of ionizing radiation or the use of IV iodinated contrast. For these reasons, it is considered the screening examination of choice: the US Preventive Services Task Force recommended a one-time screening for AAA with US in men of 65-75 years of age who have a history of smoking and for men 65-75 years of age who do not have a history of smoking [5]. Moreover, it can be used in adjunction to conventional cross-sectional imaging studies (CT, MRI) in the preoperative evaluation and post-operative surveillance of visceral vessels potentially affected by aortic procedures. For example, evaluation of branch vessel stenosis with $\mathrm{CT}$ angiography can be limited by metallic artefact due to radiopaque markers used for correct deployment of the devices: in these cases, US Doppler findings are evaluated in conjunction with CTA for proper surveillance.

The possible drawbacks of US are operator dependency and technical limitation in the examination of obese patients and in the presence of bowel gas. Furthermore, US has limited reliability in detecting morphologic alterations such as stent fracture or migration, and it has inferior sensitivity and specificity in identifying endoleaks [6].

US examination is performed with the patient in a supine position; the $\mathrm{C} 2-\mathrm{C} 5$ curved transducer is commonly used, with frequency varying upon body habitus $(2-4.5 \mathrm{MHz})$. The transducer is placed in the midline and the abdomen scanned in both longitudinal and sagittal planes. For Doppler evaluation, the smallest angle of insonation should be used and should always be $<60^{\circ}$, as it affects the measurements of peak systolic velocity. It is of fundamental importance that the Doppler examination be performed so that it may be easily reproduced, in order to reliably compare results and not to attribute velocity variations between studies to disease progression.

In patients undergoing aortic surgery, US examination focuses on the abdominal aorta and renal/mesenteric vessels (i.e. celiac artery, superior mesenteric artery). The same principles for US evaluation apply both to preoperative evaluation and post-operative surveillance of stent grafts; it must be noted that stents are not always discretely identified, depending mainly on patient characteristics and vessel anatomy.

After anatomical and morpho-dimensional evaluation of vascular structures, the first step in US evaluation is the use of colour Doppler for the correct identification of the vessels and for sample volume placement in spectral Doppler analysis. Morphology of spectral Doppler waveform is then evaluated, and peak systolic velocity (PSV) and end-diastolic velocity (EDV) are measured. Doppler waveforms vary depending upon the type of visceral vessel investigated: celiac artery (CA), renal arteries (RA) and superior mesenteric artery (SMA) in 
the postprandial phase are low-resistance vessels (resistive index [RI] of $0.55-0.7$, with wider normal ranges of $0.55-0.81$ reported for hepatic artery), while SMA in fasting state and inferior mesenteric artery (IMA) are high-resistance vessels (RI >0.7). Celiac artery and SMA resistance may be affected also by anatomic variants, occurring in up to $20 \%$ of individuals: hepatic artery branch arising from SMA is the most common and may explain a low-resistance waveform in the SMA during fasting state.

\subsubsection{Aorta}

The abdominal aorta should be evaluated both in transverse and sagittal planes, in order to measure the external diameter (outer wall to outer wall) at its widest point; any thrombus apposition or calcification should be described. Then, spectral Doppler waveform is evaluated with PSV-EDV measurements using the smallest possible angle of insonation (always $<60^{\circ}$ ), as previously mentioned.

\subsubsection{CA, SMA and IMA}

Celiac and mesenteric vessels arise from the anterior aspect of the aorta and are best imaged in the sagittal plane; conversely, hepatic artery (HA) and splenic artery (SA) are best evaluated in the transverse plane. For each vessel, spectral Doppler velocities should be sampled proximally, at the mid and distal tract.

Several velocity cut-offs have been proposed in scientific literature to diagnose arterial stenosis in native mesenteric vessels. PSV $>275 \mathrm{~cm} / \mathrm{s}$ for SMA and PSV $>200 \mathrm{~cm} / \mathrm{s}$ for CA can be used to accurately diagnose vessel stenosis $>70 \%$ [7]. IMA is not routinely evaluated, as the presence of stenosis has generally limited clinical relevance; PSV $>250 \mathrm{~cm} / \mathrm{s}$ can be used to diagnose $>50 \%$ stenosis and PSV $>270$ to predict $>70 \%$ stenosis accurately [8]. Conversely, there are no accepted criteria to diagnose pathologic stenosis in aortic branches after surgery/interventional procedures, with evidence that the cut-off used to predict stenosis in native vessels overestimates stenosis in stented/prosthetic vessels. The most plausible explanation is that the stents may reduce vessel wall compliance and may alter velocities, even in the absence of true stenosis. Proposed criteria for post-operative stenosis are PSV $>325 \mathrm{~cm} / \mathrm{s}$ for $>50 \%$ stenosis in stented SMA and PSV $>274 \mathrm{~cm} / \mathrm{s}$ in CA [9]. A valuable approach would be to evaluate changes over time by performing an examination soon after the surgical procedure in order to establish baseline parameters upon which to compare subsequent evaluations.

\subsubsection{Renal Arteries}

Correct evaluation of renal arteries can be difficult and time-consuming, even for experienced operators. RA usually arise $1-2 \mathrm{~cm}$ below the SMA, from the anterolateral (right RA) or posterolateral (left RA) aspect of the aorta. With an anterior approach, it could be difficult to obtain a correct angle of insonation for spectral Doppler analysis, as RA course parallel to the ultrasound beam; a lateral approach may thus be indicated. Accessory renal arteries, when present, can be missed due to their small size. Spectral Doppler analysis should be performed at the origin, proximal, mid and distal tract of the vessel to evaluate PSV, ESV and flow anomalies. In the presence of a stent, velocity measurements are obtained also within the stent.

Normal renal arteries are low-resistance vessels, with typical waveform and persistence of diastolic flow. Normal RA PSV is $<180 \mathrm{~cm} / \mathrm{s}$; a cut-off velocity of $>200 \mathrm{~cm} / \mathrm{s}$ is generally accepted for diagnosis of RA stenosis $>50 \%$. Other clues to vessel stenosis are increased poststenotic turbulent flow, increased aorto-renal ratio (RAR >3.5) with marked reduction of flow velocity at the distal segment and tardus-parvus pattern waveform in segmental arteries [10]. Other parameters that can be evaluated at this level are acceleration time, acceleration index and early systolic peak. Arterial velocities are usually combined with Doppler analysis of segmental arteries that allow evaluation of renovascular resistances; parenchymal damage 
with $\mathrm{RI}>0.8$ is associated with fewer functional benefits after revascularization therapy [11].

Also for renal arteries, there are no accepted criteria for the diagnosis of stenosis in stented or prosthetic arteries. A proposed criterion for renal artery stenosis $>60 \%$ in patients treated with fenestrated stent grafts consisted of PSV $>280 \mathrm{~cm} / \mathrm{s}$ and RAR >4.5 [12]. Again, the best approach would be to perform an early examination after the surgical procedure in order to establish baseline parameters upon which to compare subsequent evaluations.

\subsubsection{Contrast-Enhanced Ultrasound (CEUS)}

CEUS is an emerging technique that uses contrast agents to improve visualization of anatomic structures and characterization of focal lesions. There are several contrast agents available, consisting in microbubbles of gas stabilized by a shell (sulphur hexafluoride with a phospholipid shell [SonoVue-Lumason], octafluoropropane with a lipid shell [Definity-Luminity] and perfluorobutane with a phospholipid shell [Sonazoid]). In vascular surgery patient settings, CEUS is believed to improve the detection of endoleaks, and some authors in literature even claim its superiority over CTA [13]. However, at the time of writing, there are no indications of CEUS in vascular evaluation, as in 2016 the Food and Drug Administration approved Lumason only for the examination of the liver in adults and paediatric patients.

\subsection{MDCT Angiography}

Historically, diagnostic vascular imaging has been performed using digital subtraction angiography (DSA) by means of intravascular catheters. The development of multidetector computed tomography (MDCT) has radically transformed the role of $\mathrm{CT}$ in vascular investigation. MDCT angiography (MDCTA) allows high spatial resolution, including nearly isotropic submillimetre voxels and rapid single- breath-hold image acquisition. MDCTA yields inherent advantages over DSA, such as noninvasiveness, outstanding extravascular anatomic depiction, superior celerity, widespread accessibility, greater affordability and lower radiation dose for patients and staff, along with multiplanar post-processing for interventional planning. Thanks to these benefits, MDCTA has largely replaced DSA in routine clinical practice for the evaluation of a newly diagnosed dilatation of the aorta (Fig. 1.1), surveillance of known aortic aneurysms and follow-up after surgical or endovascular treatment [3]. Other disorders affecting the aortoiliac system may be evaluated
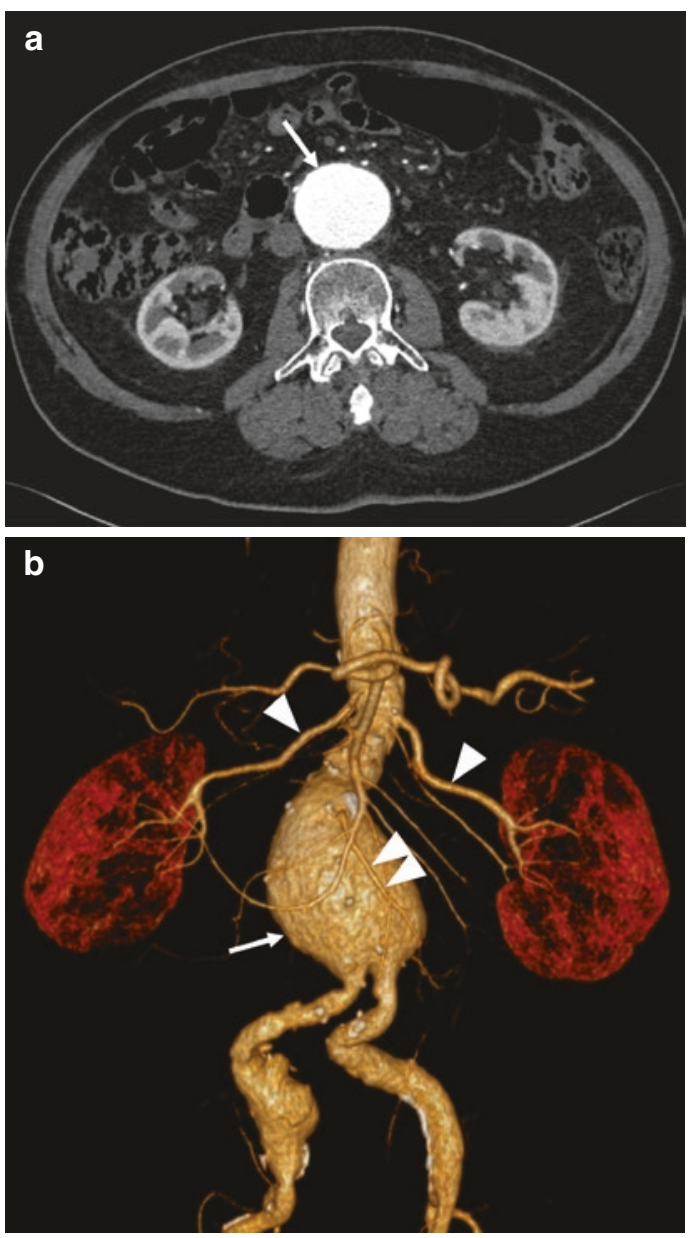

Fig. 1.1 Axial CT image (a) and volume-rendered 3D image (b) demonstrate a saccular infrarenal AAA (arrow). Note the distance between aneurysm sac and renal arteries (arrowhead). Double arrowhead IMA 
by this technique, including atherosclerotic disease, dissection, intramural hematoma, penetrating ulcer, post-traumatic vascular injury and vasculitides.

Several technical issues are supposed to be considered when performing CTA. Inclusion of a non-contrast CT scan is mandatory for imaging suspected aortic syndrome since intramural hematomas are more evident without intraarterial contrast enhancement. In the same way, calcifications are best depicted in non-contrast CT scan because of the high tissue contrast between calcium and the surrounding unenhanced tissue. Intravenous injection of iodinated contrast material is required for the intravascular opacification in CTA and has to be carefully optimized considering timing, amount and injection rate.

Injection rate actually concerns how fast the iodinated contrast agent is delivered to the vascular territory of interest. Visceral organ enhancement is relatively independent of flow rates; on the opposite, angiographic opacification heavily relies on contrast material flow and varies directly with flow rates [14]. CTA is usually performed with flow rates of $4-6 \mathrm{~mL} / \mathrm{s}$ to balance a strong peak vascular enhancement with practical considerations regarding venous access, as flow rates above $5-6 \mathrm{~mL} / \mathrm{s}$ become difficult to achieve with conventional peripheral cannulas [15]. Performing a flush with $20-30 \mathrm{~mL}$ of saline at the same injection rate as that of the contrast bolus pushes the latter from the upper extremity venous system into the central circulating blood volume. This procedure also increases the length of peak aortic enhancement and decreases the total amount of the injected contrast required for adequate aortic enhancement by 10-15 mL [14].

Due to the fact that each patient presents different circulatory times, there is a substantial risk of scanning the subject too early (with an insufficient amount of contrast material in the vessels) or too late (with undesirable venous opacification during the arterial phase) when using a fixed delay time. Therefore, synchronizing scan acquisition with contrast material delivery to the vascular territory of interest is another critical point of the CTA examination. Contrast appearance time is most commonly assessed by one of two methods. The arrival time of a test bolus of 10-20 mL administered at flow rates similar to the main contrast bolus used during MDCTA acquisition, such as $5-6 \mathrm{~mL} / \mathrm{s}$, can be assessed in order to accurately calculate the time-to-peak enhancement, so as to customize the delay time during the main contrast bolus. In the automated bolus tracking technique, a region of interest is placed over a certain location by the operator, so that the scan is initiated when the appropriate threshold for vascular enhancement is reached.

Another variable that affects contrast material administration is patient body weight. Indeed, if a fixed amount of contrast material is used, some subjects will receive an insufficient dose of contrast material (with inadequate opacification of the vessels), and at the same time, others will receive an excessive dose of contrast (which would be spared to reduce the cost of the CT examination). The overall contrast volume should be approximately equal to the injection rate (in $\mathrm{mL} / \mathrm{s}$ ) multiplied by the scan duration in second plus 5-10 s: typical contrast volumes range from 60 to $120 \mathrm{~mL}$ [16].

After the acquisition of the arterial phase, a portal venous phase is usually obtained with a 60-70 s delay time from the beginning of contrast material infusion in order to evaluate for late filling of potential false lumen in dissections, slow endoleaks in endovascular stent repair (Fig. 1.2), contrast extravasation from aortic rupture (Fig. 1.3) or inflammatory enhancement in vasculitides and infections.

Digital image post-processing of the acquired data is performed using various algorithms, including maximum intensity projection (MIP), volume rendering (VR) and multiplanar reconstruction (MPR) techniques (Fig. 1.4). VR provides us a topographic display of the aortoiliac system and visceral arterial branches, by means of an exquisite immersive visualization for browsing the $3 \mathrm{D}$ volume and displaying vascular pathology as well. MIP techniques are two-dimensional representations of three-dimensional data that yield angiography-like images with an excellent overview of vascular anatomy. They require viewing from different angles for a three-dimensional per- 

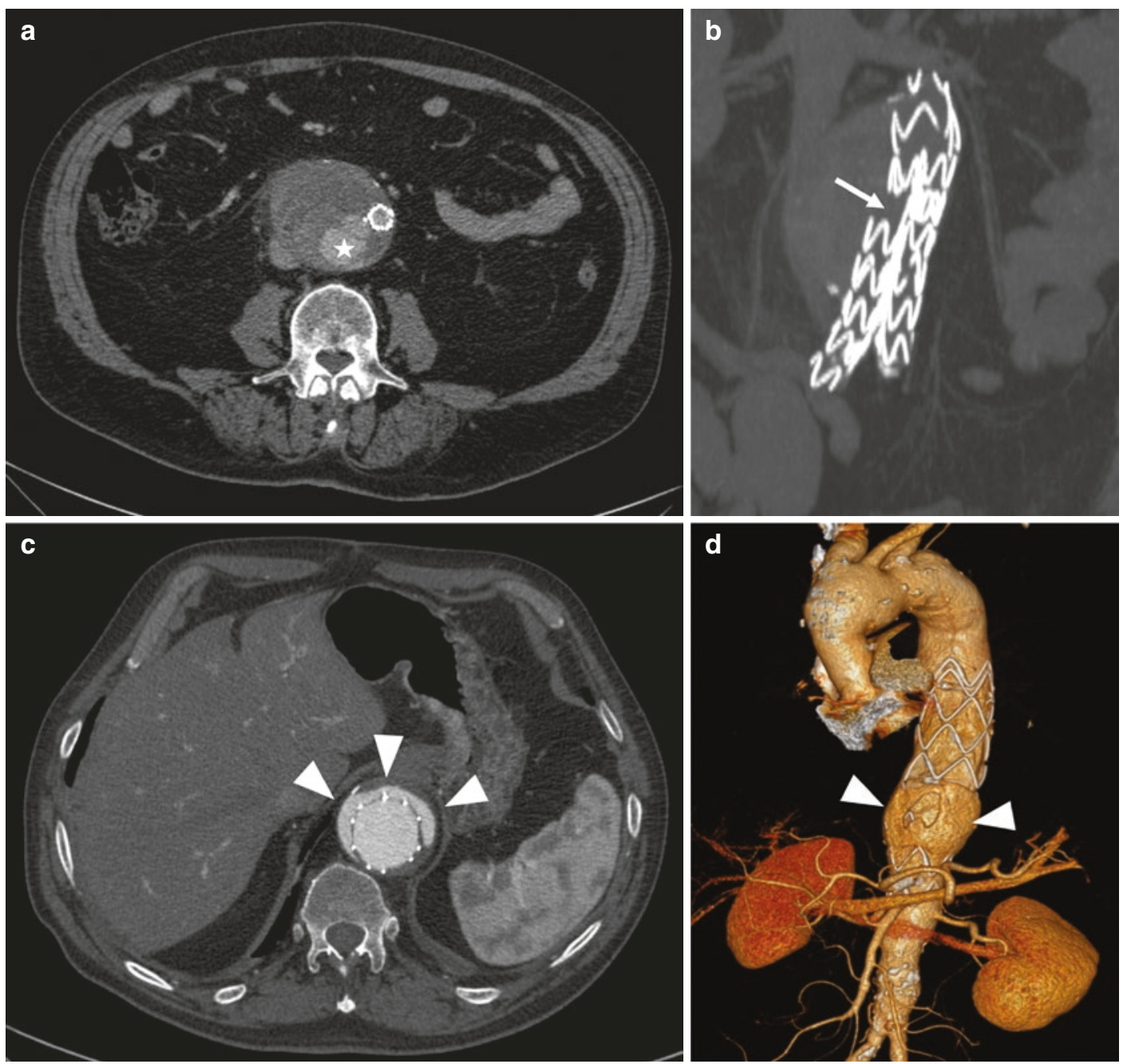

Fig. 1.2 (a, b) 80-year-old man with a progressively enlarging false lumen due to endoleak after endovascular aortic repair for AAA. Axial CT image (a) shows the enhancing false lumen (star) external to the stent graft at the level of the right iliac branch. MIP reconstruction (b) demonstrates discontinuity (arrow) of the metal prosthesis

spective. MIP is limited in the evaluation of vascular stenosis because of the superimposition of high-attenuating vascular calcifications over the vessel lumen. In these circumstances, multiplanar reformations can display inner lumen and outer mural calcification and can accurately portray the degree of arterial stenosis. Ideally, the assessment of CTA datasets is best accomplished through a two-step process of standard axial image interpretation and subsequent 3D image review. at the origin of the right iliac stent-graft branch. (c, d) 69-year-old man with endoleak after endovascular repair for TAAA. Axial CT image (c) and VR image (d) show contrast material accumulation (arrowheads) in the false lumen external to stent graft

Many patients undergoing evaluation of the aorta will need serial imaging follow-up over many years for monitoring aneurysm size, extent of dissection and possible post-procedural complications. Multiple strategies have been introduced in MDCTA in an attempt to reduce patient radiation dose without degradation of image quality. With automatic tube current modulation, the tube current is by default reduced when scanning low-attenuating regions and increased for areas of higher atten- 


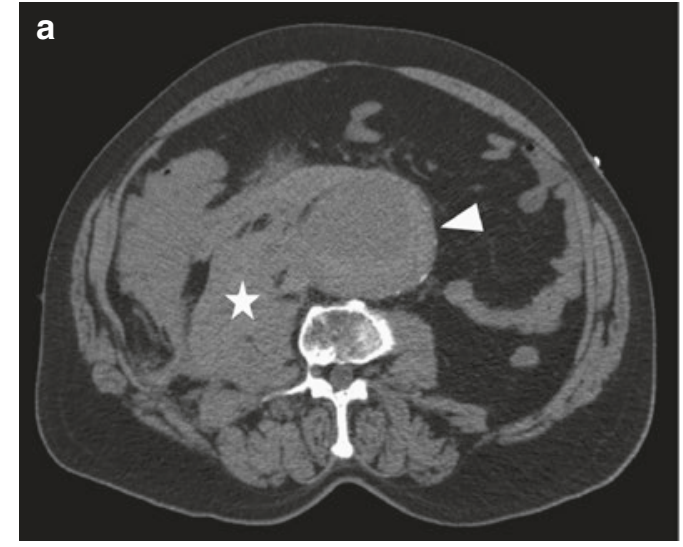

Fig. 1.3 Axial non-contrast image from CTA (a) demonstrates a large retroperitoneal hematoma (star) and AAA (arrowhead), compatible with ruptured AAA. Axial contrast-enhanced image (b) demonstrates irregularity along

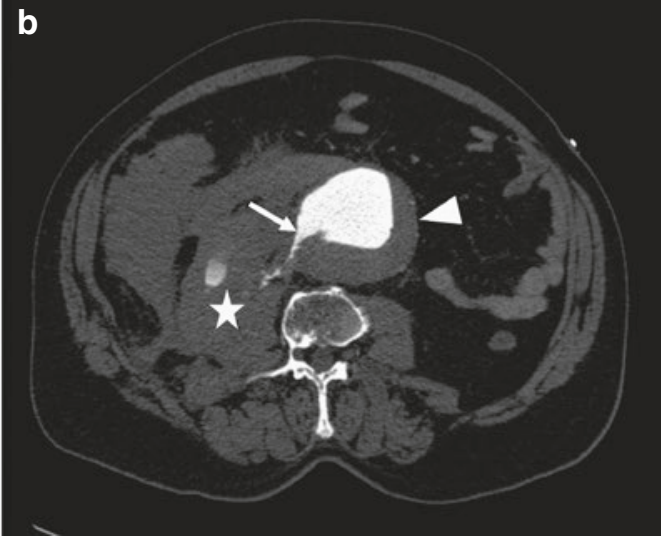

the right lateral wall of the aorta with active extravasation of contrast material into the hematoma (arrow), confirming exact site of AAA rupture

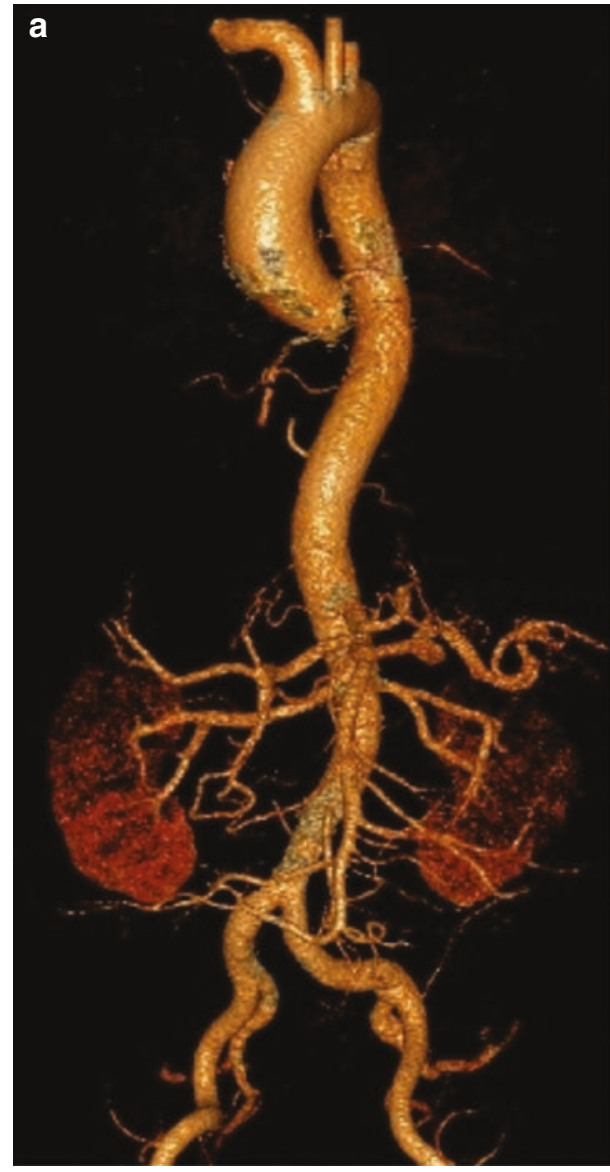

Fig. 1.4 (a) Volume-rendered 3D image in a coronal projection, after bone editing, shows the normal anatomy of the aorta and its branches. (b) Axial contrast-enhanced image shows the origin of renal arteries (arrows). (c) Coronal MIP rendering demonstrates a complete map of
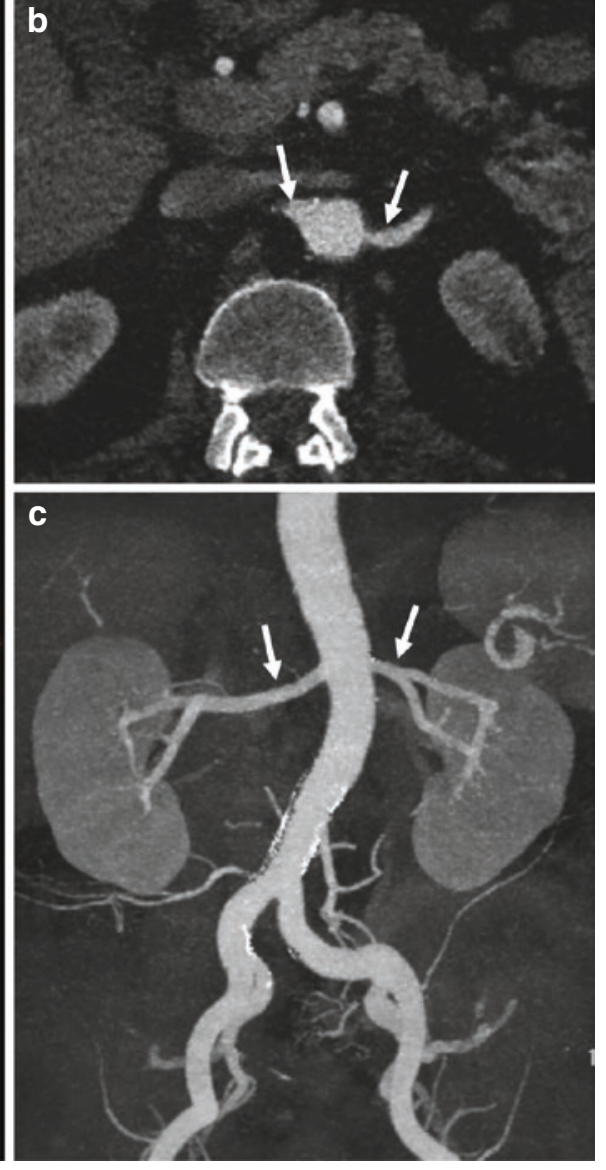

the renal arteries (arrows) in one image, but threedimensional relationship is not maintained with MIP renderings. (d) MPR of the right renal artery: this technique is useful for elongating a tortuous structure in order to identify the location of maximal vessel dilation or narrowing 


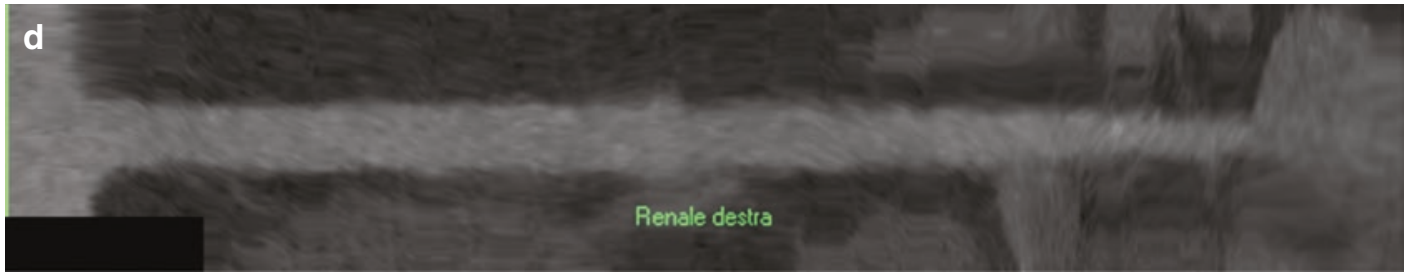

Fig. 1.4 (contineud)
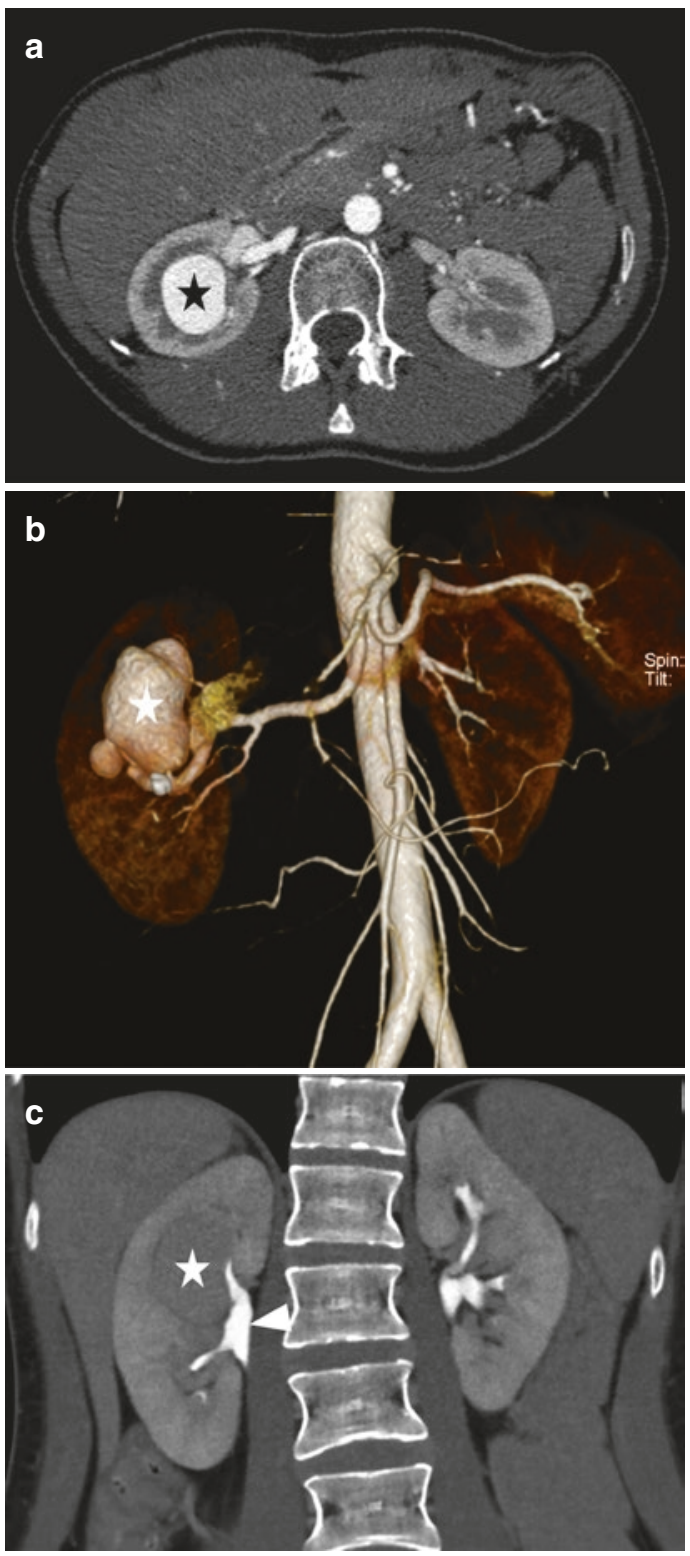

Fig. 1.5 Axial contrast-enhanced image (a) and VR image (b) show a big saccular aneurysm (star) arising from an intrarenal branch of the right renal artery. Delayed coronal CT image (c) delineates the relationship of the aneurysm sac (star) to the renal pelvis (arrowhead) uation. Iterative reconstruction algorithms allow for radiation dose reduction by improving the signal-to-noise ratio at lower tube current levels [17].

Thanks to its rapid acquisition of CT volumes during peak arterial enhancement and isotropic resolution, MDCTA permits the visualization of smaller arterial structures, such as abdominal visceral branches, becoming a useful tool to study normal and variant anatomy as well as pathologic conditions of the visceral vasculature (Figs. 1.5 and 1.6). For example, CT angiography is helpful in the evaluation of the renal arteries regarding stenosis, vascular mapping in healthy living renal donors and transplant recipients as well as vascular complications after renal transplantation. In this case, MDCT angiography especially focuses on renal artery anatomy with emphasis on possible variations that may hamper kidney transplantation, such as early branching or accessory renal arteries. These may arise from vessels as far distant as the common iliac artery, so that failure to detect such vessels may cause partial post-transplant infarction.

\subsection{Magnetic Resonance Angiography}

Nowadays, magnetic resonance angiography (MRA) has become a good alternative to the imaging modalities listed above, allowing a noninvasive depiction of blood vessels, with reasonable timing, mainly due to great technological developments (such as parallel imaging, phasedarray coils and fast slew rate). Furthermore, MR angiography uses radiofrequencies combined with magnetic fields instead of ionizing radiations, which is safer for the patient [18]. 

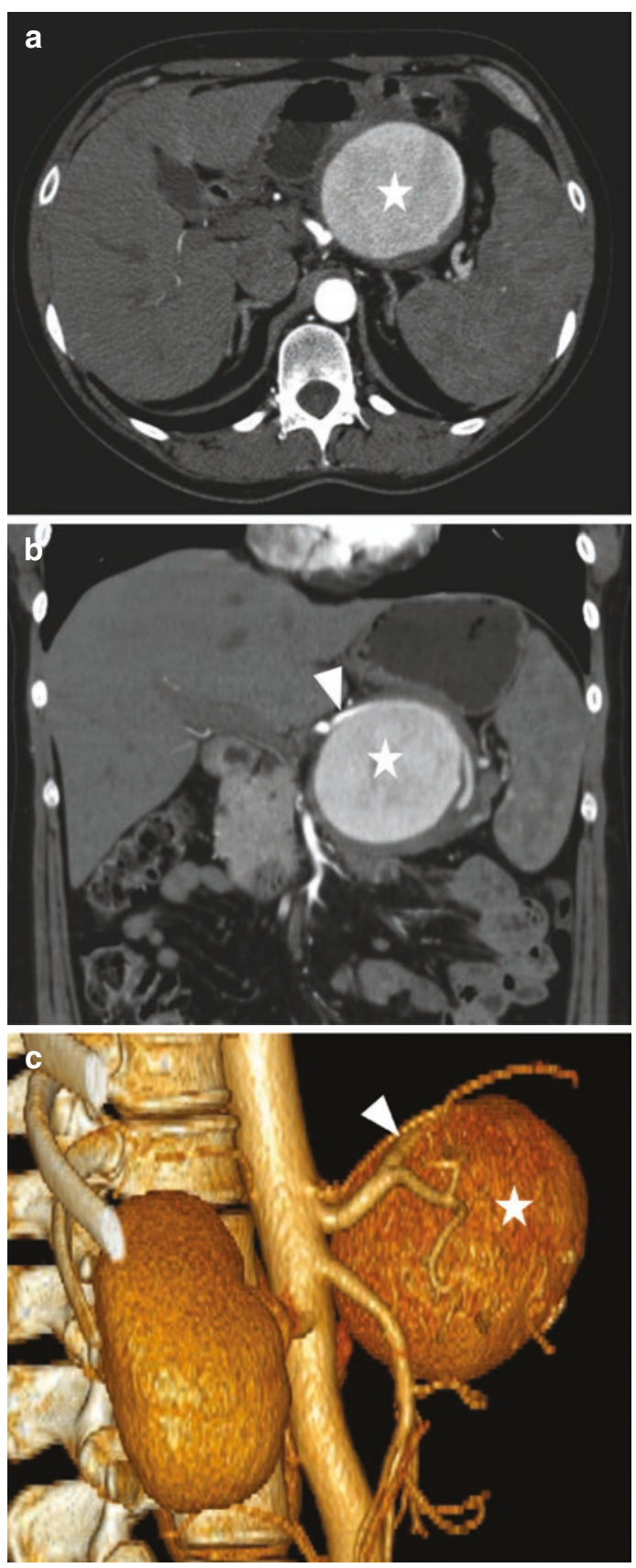

Fig. 1.6 Axial (a) and coronal (b) CT images show a large enhancing aneurysm (star) arising from the splenic artery (arrowhead). VR image (c) again shows the large splenic artery aneurysm (star) and better defines its relationship with splenic artery (arrowhead)
MR angiography also allows for the quantification of blood flows in terms of direction and velocity. Datasets can be acquired in $3 \mathrm{D}$ or as stacks of 2D images, containing all the vessels in the volume of interest. When $3 \mathrm{D}$ datasets are acquired, post-processing can obtain reconstruction in any projection.

MRA sequences are mainly divided into two groups: contrast and non-contrast MR angiography (Fig. 1.7). Acquiring MRA without the need for contrast injection is extremely useful, especially in patients with impaired renal function. Nevertheless, when contrast agent is used, it is usually a gadolinium chelate, always 'safer' compared to iodinated contrast agents.

MRA has the incredible added value of providing information about vessels, but not only: thanks to the MR imaging ability of characterizing tissues, detailed information can be obtained even regarding the vessel walls (Fig. 1.8) and thrombi and, in general, about surrounding tissues.

\subsubsection{Non-contrast MR Angiography (Flow-Based MRA)}

Blood flows often create artefacts which can influence and weaken image quality. On the other hand, these same artefacts can be used to develop noninvasive imaging techniques with no need for contrast agent injection [19]. There are few main 'flow effect' categories which must be cited because they can be advantageously used to obtain MRA sequences:

- 'Amplitude effect' or time of flight (TOF) takes advantage from blood proton flow inside a volume of interest, where surrounding static tissue have been saturated so that they cannot produce signal. Therefore, only moving protons, specifically, the protons of blood subject to a movement, will generate signal. 

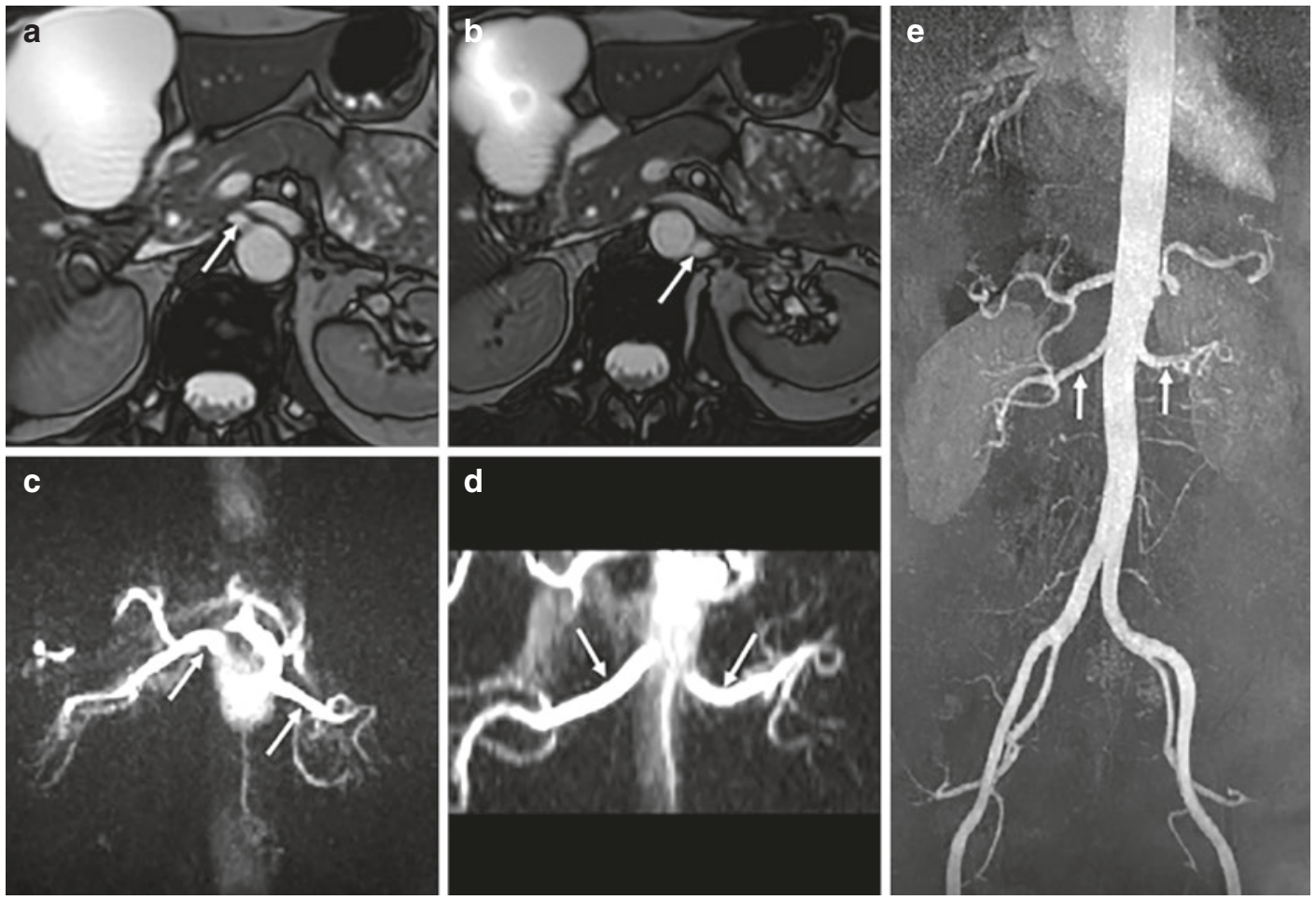

Fig. 1.7 Axial-balanced MR images $(\mathbf{a}, \mathbf{b})$ show the origin of the left and right renal arteries (arrow). Axial (c) and coronal (d) MIP reconstructions of phase-contrast images clearly depict both renal arteries without contrast

medium administration. MIP reconstruction of images obtained during administration of contrast medium (e) renal arteries is indicated by arrows
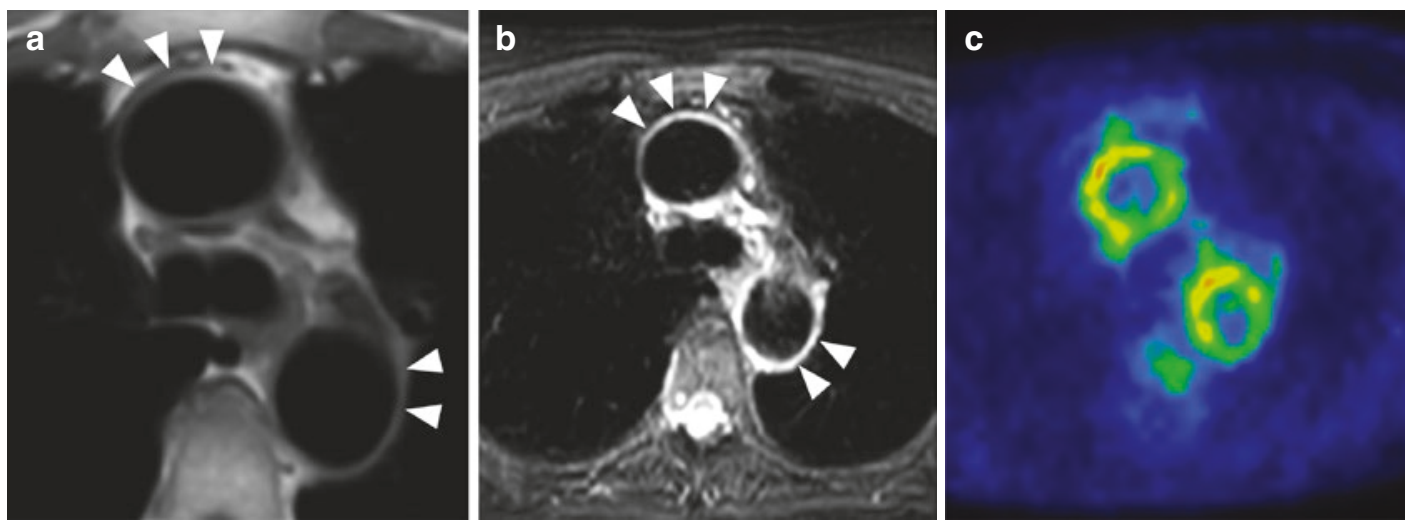

Fig. 1.8 50-year-old female with giant cell arteritis. Proton density (PD) image (a) shows arterial wall thickening (arrowheads). Arterial wall is hyperintense in
STIR MR image (b) indicating the presence of oedema and inflammation. FDG-PET study (c) depicts increased metabolic activity of the vessel wall 
- Phase-contrast effect (PC) takes advantage of the detection of changes in phase of the transverse magnetization of blood protons flowing along the direction of a magnetic field compared to stationary protons.

- The so-called signal void phenomenon is something to be aware of: it consists in the lack of signal from protons moving outside the field of view (which is the area we are studying). On the contrary, when blood flow is turbulent, protons can move back to the voxel where they originally were giving signal.

Different non-contrast MRA techniques are now listed:

\section{Time-of-flight (TOF) MRA:}

Usually consists of a gradient echo sequence, optimized to maximize signal of vascular structures compared to the one of other anatomical structures, saturating signal from surrounding stationary tissue. Fat saturation and selective water content saturation can also help to improve signal. TOF MRA takes advantage from the 'entry slice phenomenon', also known as 'inflow enhancement', which refers to the gain of signal coming from blood protons entering the voxel.

Signal intensity is directly proportional to flow velocity and is related to vessel length and orientation. Signal will be higher if the sequence is acquired perpendicular to the major axis of the vessel.

This sequence does not allow to differentiate arterial from venous flow; therefore, the two can sometimes appear overlapped. To selectively image venous or arterial flows, TOF MRA can take advantage from the opposite flow direction of these two vascular structures, which can be differentiated using added selective saturation pulses.

TOF MRA can be:

- 2D (sequential, multislice), which consists in the sequential acquisition of single thin sections. 2D is more sensitive to slow flows and useful for venous studies. 2D TOF can also overcome respiratory motion, allowing acquisi- tion of single or few breath-held slices at a time. The main limit of these 2D sequences is their low spatial resolution due to medium-high slice thickness compared to 3D technique [20].

- 3D (volume slab) consists in the contemporary acquisition of data from a single volume, successively subdivided into several thin slices. 3D has good spatial resolution and good signal-to-noise ratio. 3D sequences also allow for the reconstruction of images in different planes, not only in the original acquisition plane. 3D is more sensitive to quick flows and small vessels. The main 3D TOF MRA limits are related to poor sensitivity to slow flows, high sensitivity to breathing artefacts and a long acquisition time [21].

TOF MRI has been widely used for vascular study of the head and neck, especially for carotids and the Willis circle.

2. Phase-contrast sequences are gradient echo sequences based on the detection of changes to the phase of moving protons inside the vessels under study, when under the effect of a bipolar gradient. The bipolar gradient generates different changes in phase, depending on whether spins are stationary or moving: when spins are stationary, their phase variation depends only on their position; when spins are moving, phase variation depends on their position, movement direction and velocity. It is therefore important to set the right parameters before acquiring these sequences, such as the right VENC (velocity encoded), to avoid artefacts related to a wrong codification of proton velocity.

The main advantage of PC is their ability to give information on position, direction and velocity of flows. To obtain a quantitative evaluation of a flow, the acquisition needs to be perpendicular to the flow direction; then, a timeresolved curve of flow velocity can be obtained. When both the time-resolved curve of flow velocity and the sectional area of the vessel are calculated, flow can be quantified [22]. 
3. 4D flows are the latest version of phasecontrast sequences: a $4 \mathrm{D}$ flow sequence is a PC with flow-encoding resolved relative to all three dimensions of space and to the dimension of time along the cardiac cycle $(3 \mathrm{D}+$ time $=4 \mathrm{D}) .4 \mathrm{D}$ flows allow retrospective placement of analysis planes at any location within the acquisition volume and calculation of blood flow through any planes of interest across the $3 \mathrm{D}$ volume. They can be used to derive time-averaged $3 \mathrm{D}$ phase-contrast MR angiography (PC-MRA). PC can be 2D or 3D as well. PC plays an important role in the study of arterial and venous malformations, as well as cardiac valvular flows [23, 24].

4. Fast imaging with steady-state precession (TrueFISP): is a sequence with a very particular high blood signal, thanks to a mixed $\mathrm{T} 2 / \mathrm{T} 1$ weight. These sequences are very quick and allow acquisition of breath-held 3D images, with prospective synchronization. They have been widely used for renal artery study. The limit is that not only blood but also fluids have high signal [25].

Main limits of non-contrast MRA are possible overlap of arterial and venous flows, multidirectionality of flows, turbulence, respiratory and cardiac movement artefacts and high fat signal.

\subsubsection{Contrast MR Angiography}

Constant improvement of hardware and software allows the development of contrast-enhanced magnetic resonance angiography (CE-MRA), which is based on intravenous administration of a bolus of paramagnetic extracellular contrast agent which significantly increases blood signal intensity during its first passage due to its ability of shortening the T1. Images are acquired during the first passage of the contrast into arteries. Vessels are imaged independently from flow type (i.e. laminar, turbulent) and velocity. CE-MRA is also unaffected by flow de-phasing, which often takes place in tortuous and stenotic vessels.
CE-MRA usually consists of a 3D gradient echo (FLASH) sequence, which is very quick and can image vessels in the arterial phase before significant venous enhancement $(<30 \mathrm{~s}$ of acquisition length).

Of fundamental importance is the correct timing of CE-MRA acquisition: images need to be coordinated with contrast arrival in the vessel of main interest. Contrast agent dose should also be limited: the dose should be kept to the minimum necessary to obtain good opacification.

All CE-MRAs require the acquisition of a precontrast scan and the angiographic scan (during the higher peak of enhancement inside the vessels) [26, 27].

CE-MRA can be differentiated according to different ways of acquiring images in relation to contrast agent injection:

1. Test bolus MRA: The angiographic sequence is preceded by a dynamic sequence acquired every few seconds on the vessel of interest during the injection of a small amount of contrast agent. After the acquisition of this sequence, a ROI is drawn on the central part of the vessel of interest, through dedicated software, to obtain the time of arrival of contrast agent, so that it can be used as the right timing for the acquisition of the full sequence.

2. Fluoroscopic MRA is a real-time triggering CE-MRA. A real-time sequence, located on the vessel of interest, is acquired during contrast injection, to show the contrast arrival into the vessel so that the sequence can be manually started when preferred according to the vessel opacification.

3. Bolus track, also called 'automated bolus detection' technique, is another real-time triggering CE-MRA. A real-time sequence, located on the vessel of interest, is acquired during contrast injection, and a ROI is positioned in the centre of the vessel. The scan automatically measures the intensity of the pixel in the ROI, so that when the predefined threshold is reached, the scan will automatically start to acquire the main sequence. 
Irrespective of the kind of sequence used, subtraction of pre- and post-contrast images should always be completed, to obtain pictures where filled vessels only are visualized. Several reconstructions are possible starting from CE-MRA.

CE-MRA has become very popular in several contexts: for evaluation of atherosclerotic pathology involving carotid arteries, thoracic and abdominal aorta, mesenteric vessels and upper and lower extremity vessels, pre-surgical planning for kidney donors and receivers, follow-up of patients undergoing surgery (i.e. bypass), study of arterial or venous malformation and study of congenital abnormalities such as aortic coarctation.

\subsubsection{Contrast Agents}

There are two main categories of contrast agents (CA) used for MRA: gadolinium (Gd)-based CA and iron oxide (IO)-based CA [28, 29].

\section{Paramagnetic gadolinium-based CA}

Gadolinium chelates are popular contrast agents, which are of main use in clinical routine considering high availability. They are paramagnetic, which means they shorten the T1 relaxivity of tissues where they are injected. They can be further subdivided according to the ability or inability to interact with intravascular proteins.

Specifically, gadolinium CA with no capacity for protein interaction are simply extracellular, interstitial and intravascular CA, excreted and unchanged through the kidneys. Examples of these CA are Gd-BDTPA (Magnevist), Gd-DOTA (Dotarem), Gd-DTPA-BMA (Omniscan) and Gd-BT-DO3A (Gadovist).

Gadolinium CA with weak protein interaction, such as the Gd-BOPTA (MultiHance) and Gd-EOB-DTPA, can weakly and transiently interact with serum proteins, specifically albumin. Therefore, they have higher T1 relaxivity which manifests as a greater intravascular signal intensity. They show good vessel enhancement up to 50-60 min after CA injection.
Finally, the so-called blood pool CA are Gd-derived CA with a prolonged and increased intravascular signal. They are composed of two types of molecules: Gd chelates with strong protein interaction, which allows them to stay for longer inside vessels, and $\mathrm{Gd} \mathrm{CA}$ with macromolecular structures whose dimension limits their extravasation.

Gadolinium safety: gadolinium has been proven to be safe, especially at low doses. A possible concern is the occurrence of nephrogenic systemic fibrosis (NSF), which is a fibrosis of unexplained origin, which has been seen in patients with impaired renal function (eGFR $<30$ ). The gadolinium retention within the body following possible transmetalation and release of free gadolinium has been hypothesized as possible cause and concern. Allergic reaction to gadolinium is possible, even though very uncommon (occurrence reported between 0.005 and $0.7 \%)$ [30].

\section{Superparamagnetic iron oxide-based CA}

These CA are based on ultrasmall IO particles (e.g. USPIO or gadofosveset trisodium, Vasovist, the latter no more in use). They strongly shorten $\mathrm{T} 1$ relaxivity, but they also have $\mathrm{T} 2 *$ effect, generating possible artefacts, especially at highcontrast doses.

\subsubsection{Multiparametric MRI: Complimentary Sequences}

An important characteristic of MR imaging is the possibility of pairing other kinds of sequences to standard MRA sequences. For example, T2-STIR sequences can be added when studying vascular pathology, especially inflammatory pathology, such as arthritis. T2 sequences are highly sensitive to the presence of oedema, which appears as hyperintensity of signal within a structure. Proton density and T1 sequences may also help characterize pathologic tissues, when present (such as thrombi, ulcer or fibrotic changes). 


\subsubsection{Reconstruction}

There are several ways to manipulate and reconstruct images to obtain the overall morphology and spatial relationship of anatomical structures:

- Multiplanar reconstruction or reformation (MPR) is the process of reformatting images from the original reconstruction plane into a different orientation, without altering the MRA voxels. They allow to visualize both vessels and surrounding structures within the same image. MPRs often consist of sagittal, axial and coronal images or images orientated according to the main axes of the vascular structure of interest. Reconstructions on oblique planes are sometimes feasible, as they are extremely useful for a proper analysis of tortuous vessels.

- Maximum intensity projection (MIP) is the most popular MRA image reconstruction, allowing a bi-dimensional representation of vessels. It produces images similar to those from conventional X-ray angiography. Firstly, the reconstruction orientation should be planned (i.e. vertical, horizontal, lateral), and then MIP are obtained casting parallel rays through the 3D dataset with each pixel of the projection image plane representing the maximum signal intensity encountered along the corresponding way. They are very useful for visualizing arterial phase of contrastenhanced MRA. On the other hand, MIP can hide some anatomical details and spatial relationship of vessels with the surroundings. Overlapping of several structures could potentially hide or mimic a significant stenosis [31].

- Volume rendering (VR) allows the visualization of all 3D data of a volume into a single 2D projection. It works on voxel intensity, creating translucent rendering of the full volumetric dataset. Usually, the operator can manually select colour, opacity and luminosity corresponding to different voxel intensities. For VR, intensity values of different structural parts of the image are given to be classified as visible or non-visible (or with a specific colour or opacity).

- Virtual intraluminal endoscopy is used to evaluate the vascular wall from the inside. It combines the feature of endoscopic viewing and cross-sectional volumetric imaging, creating a series of perspective views calculated from points inside the vasculature. These views can then be reconstructed with surface or volume rendering. This technique is not routinely used, because of its poor diagnostic advantages, despite being an advanced technique.

\section{References}

1. Berko NS, Jain VR, Godelman A, Stein EG, Ghosh S, Haramati LB. Variants and anomalies of thoracic vasculature on computed tomographic angiography in adults. J Comput Assist Tomogr. 2009;33:523-8.

2. Liu PS, Platt JF. CT angiography of the renal circulation. Radiol Clin N Am. 2010;48:347-65.

3. Budovec JJ, Pollema M, Grogan M. Update on multidetector computed tomography angiography of the abdominal aorta. Radiol Clin N Am. 2010;48:283-309.

4. Ugurel MS, Battal B, Bozlar U, Nural MS, Tasar M, Ors F, Saglam M, Karademir I. Anatomical variations of hepatic arterial system, coeliac trunk and renal arteries: an analysis with multidetector CT angiography. Br J Radiol. 2010;83:661-7.

5. Guirguis-Blake JM, Beil TL, Senger CA, Whitlock EP. Ultrasonography screening for abdominal aortic aneurysms: a systematic evidence review for the U.S. preventive services task force. Ann Intern Med. 2014;160:321-9.

6. Raman KG, Missig-Carroll N, Richardson T, Muluk SC, Makaroun MS. Color-flow duplex ultrasound scan versus computed tomographic scan in the surveillance of endovascular aneurysm repair. J Vasc Surg. 2003;38(4):645-51.

7. Moneta GL, Lee RW, Yeager RA, Taylor LM, Porter JM. Mesenteric duplex scanning: a blinded prospective study. J Vasc Surg. 1993;17:79-86.

8. Pellerito JS, Revzin MV, Tsang JC, Greben CR, Naidich JB. Doppler sonographic criteria for the diagnosis of inferior mesenteric artery stenosis. J Ultrasound Med. 2009;28:641-50.

9. Aburahma AF, Mousa AY, Stone PA, Hass SM, Dean LS, Keiffer T. Duplex velocity criteria for native celiac/superior mesenteric artery stenosis vs in-stent stenosis. J Vasc Surg. 2012;55:730-8. 
10. Olin JW, Piedmonte MR, Young JR, DeAnna S, Grubb M, Childs MB. The utility of duplex ultrasound scanning of the renal arteries for diagnosing significant renal artery stenosis. Ann Intern Med. 1995; 122:833-8.

11. Radermacher J, Chavan A, Bleck J, et al. Use of Doppler ultrasonography to predict the outcome of therapy for renal-artery stenosis. N Engl J Med. 2001;344:410-7.

12. Mohabbat W, Greenberg RK, Mastracci TM, Cury M, Morales JP, Hernandez AV. Revised duplex criteria and outcomes for renal stents and stent grafts following endovascular repair of juxtarenal and thoracoabdominal aneurysms. J Vasc Surg. 2009;49:827-37.

13. Gürtler VM, Sommer WH, Meimarakis G, Kopp R, Weidenhagen R, Reiser MF, Clevert DA. A comparison between contrast-enhanced ultrasound imaging and multislice computed tomography in detecting and classifying endoleaks in the follow-up after endovascular aneurysm repair. J Vasc Surg. 2013;58:340-5.

14. Bae KT. Intravenous contrast medium administration and scan timing at CT: considerations and approaches. Radiology. 2010;256:32-61.

15. Fleischmann D. CT angiography: injection and acquisition technique. Radiol Clin N Am. 2010;48:237-47.

16. Scheske JA, Chung JH, Abbara S, Ghoshhajra BB. Computed tomography angiography of the thoracic aorta. Radiol Clin N Am. 2016;54:13-33.

17. Hara AK, Paden RG, Silva AC, Kujak JL, Lawder HJ, Pavlicek W. Iterative reconstruction technique for reducing body radiation dose at CT: feasibility study. Am J Roentgenol. 2009;193:764-71.

18. Schneider G, Prince MR, Meaney JFM, Ho VB. Magnetic resonance angiography. Berlin: Springer; 2005. p. 358.

19. Korosec FR, Mistretta CA. MR angiography: basic principles and theory. Magn Reson Imaging Clin N Am. 1998;6:223-56.

20. Keller PJ, Drayer BP, Fram EK, Williams KD, Dumoulin CL, Souza SP. MR angiography with two-dimensional acquisition and threedimensional display. Work in progress. Radiology. 1989;173:527-32.

21. Laub GA, Kaiser WA. MR angiography with gradient motion refocusing. J Comput Assist Tomogr. 1988;12:377-82.

22. Laub G, Gaa J, Drobnitzky M. Magnetic resonance angiography techniques. Electromedica. 1998;66:68-75.

23. Dyverfeldt P, Bissell M, Barker AJ, et al. 4D flow cardiovascular magnetic resonance consensus statement. J Cardiovasc Magn Reson. 2015;17:72.

24. Vasanawala SS, Hanneman K, Alley MT, Hsiao A. Congenital heart disease assessment with $4 \mathrm{D}$ flow MRI. J Magn Reson Imaging. 2015;42:870-86.

25. Deshpande VS, Shea SM, Laub G, Simonetti OP, Finn JP, Li D. 3D magnetization-prepared true-FISP: a new technique for imaging coronary arteries. Magn Reson Med. 2001;46:494-502.

26. Lohan DG, Saleh R, Nael K, Krishnam M, Finn JP. Contrast-enhanced MRA versus nonenhanced MRA: pros and cons. Appl Radiol. 2007;36:3-15.

27. Lin J, Li D, Yan F. High-resolution 3D contrastenhanced MRA with parallel imaging techniques before endovascular interventional treatment of arterial stenosis. Vasc Med. 2009;14:305-11.

28. Kirchin M, Runge V. Contrast agents for magnetic resonance imaging: safety update. Top Magn Reson Imaging. 2003;14:426-35.

29. Laurent S, Vander EL, Muller RN. Comparative study of the physicochemical properties of six clinical low molecular weight gadolinium contrast agents. Contrast Media Mol Imaging. 2006;1:128-37.

30. Runge VM. Safety of magnetic resonance contrast media. Top Magn Reson Imaging. 2001;12:309-14.

31. Calhoun PS, Kuszyk BS, Heath DG, Carley JC, Fishman EK. Three-dimensional volume rendering of spiral CT data: theory and method. RadioGraphics. 1999;19:745-64. 